\title{
SI Traceable Solar Spectral Irradiance Measurement Based on a Quantum Benchmark: A Prototype Design
}

\author{
Xiaobing Zheng *, Maopeng Xia, Wenchao Zhai, Youbo Hu, Jianjun Li, Yinlin Yuan and \\ Weiwei Pang \\ Key Laboratory of Optical Calibration and Characterization, Anhui Institute of Optics and Fine Mechanics, \\ Chinese Academy of Sciences, Hefei, Anhui 230031, China; mpxia@aiofm.ac.cn (M.X.); \\ wczhai@aiofm.ac.cn (W.Z.); youbohu@aiofm.ac.cn (Y.H.); jjli@aiofm.ac.cn (J.L.); ylyuan@aiofm.ac.cn (Y.Y.); \\ wwpang@aiofm.ac.cn (W.P.) \\ * Correspondence: xbzheng@aiofm.ac.cn
}

Received: 1 March 2020; Accepted: 29 April 2020; Published: 4 May 2020

\begin{abstract}
We propose a space benchmark sensor with onboard SI (Système International) traceability by means of quantum optical radiometry. Correlated photon pairs generated by spontaneous parametric down-conversion (SPDC) in nonlinear crystals are used to calibrate the absolute responsivity of a solar observing radiometer. The calibration is systematic, insensitive to degradation and independent of external radiometric standards. Solar spectral irradiance at 380-2500 nm is traceable to the photon rate and Planck's constant with an expected uncertainty of about $0.35 \%$. The principle of SPDC calibration and a prototype design of the solar radiometer are introduced. The uncertainty budget is analyzed in consideration of errors arising from calibration and observation modes.
\end{abstract}

Keywords: radiometric benchmark; spontaneous parametric down-conversion; correlated photons; detector calibration; solar irradiance

\section{Introduction}

Space radiometric benchmarks were proposed in recent years to reveal the trend of climate change and evaluate the energy budget of the Earth system with high confidence [1-4]. Benchmark sensors can also serve as on-orbit references and inter-calibrate other operational sensors by simultaneous nadir observation of certain targets, and improve data consistency between different missions. The primary challenge to fulfil a benchmark mission is SI-traceable radiometry on orbit and high accuracy over decadal time period.

Space benchmark sensors are expected to measure solar total irradiance, solar spectral irradiance and Earth reflective radiance with uncertainties of about $0.02 \%, 0.2 \%$ and $1 \%$, respectively [1-4], comparable to the highest accuracies currently achievable in national metrology labs. These stringent requirements of uncertainties cannot be satisfied with traditional traceability chains connecting space sensors to laboratory radiometric standards [3]. In the past 40 years, in-flight calibration techniques such as vicarious and onboard calibration [5] have been developed to correct inevitable sensor degradation in space. Vicarious calibration calculates sensor received radiance by measuring ground reflected radiation and modeling atmospheric transmittance at test sites and satellite overflight moments. Improvement of its accuracy is limited by nonuniform reflection of test sites, and uncontrollable atmospheric properties along the propagation path from test site to the satellite. Onboard calibration aims to monitor the change of sensor responsivity by dedicated light sources or reference detectors. However, its stability is subject to degradation of the onboard calibrator itself [6,7] and it is doubtful to meet the ultrahigh accuracies required for climate observation. Several approaches have been 
developed to monitor and assess the degradation of space-borne solar instruments and there is no single best method, but rather a combination of methods taking into account the applicability to the mission targets and the instrumental design [8]. In another respect, operating an onboard calibrator with climate accuracy for every sensor may result in unacceptable engineering complexity and expenses. Scale inconsistency between different vicarious calibrations or onboard calibrations could be another source of uncertainty due to different tracing routes to different radiometric standards. Despite all efforts in the past, there is still an accuracy gap of about one order of magnitude between the current operational calibration and a benchmark for climate research $[1,3]$.

Extensive efforts have been made to realize a space radiometric benchmark without traceability to ground metrological standard facilities. An electrical substitution radiometer is already used as an onboard standard $[9,10]$ which converts optical flux measurement to electrical heating power measurement with higher accuracy and repeatability. Comparing with standard light sources or detectors, physical constants are more suitable for space benchmarking since they are invariant in the space environment and in no need of correction for degradation. For example, transient temperature during the phase change of certain material is used to calibrate temperature sensors of an onboard blackbody [11].

In this paper a nonlinear optical effect, i.e., spontaneous parametric down-conversion (SPDC), is proposed as a new attempt for space benchmarking. The working principle of SPDC-based absolute calibration and an SPDC-calibrated radiometer are introduced in Sections 2 and 3, respectively. The goal is to realize an uncertainty of about $0.35 \%$ for solar spectral irradiance measurements at visible to shortwave infrared spectrum. Uncertainty sources are discussed in Section 4 and an initial evaluation of the uncertainty budget is presented to show the potential of the instrument.

\section{Principle of SPDC-based Absolute Calibration}

SPDC is a quantum optical effect occurring in a nonlinear crystal pumped by a laser beam. Since the 1960s, theories of SPDC have been developed [12,13] and important applications emerged in the fields of quantum communication [14] and quantum radiometry [15].

In a SPDC process, pumping photons interact with quantum vacuum noise in crystal and their energy is coupled into pairs of photons with lower frequencies (down-conversion). As shown in Figure 1, one pumping photon has a certain possibility (efficiency) to decay into a pair of photons, usually called correlated or entangled photons, since they have definite relations between their frequencies, directions and polarizations. Detection of one photon and its properties indicates the existence of the correlated photon and the corresponding properties. This exceptional feature forms the physical basis for our benchmark instrument.

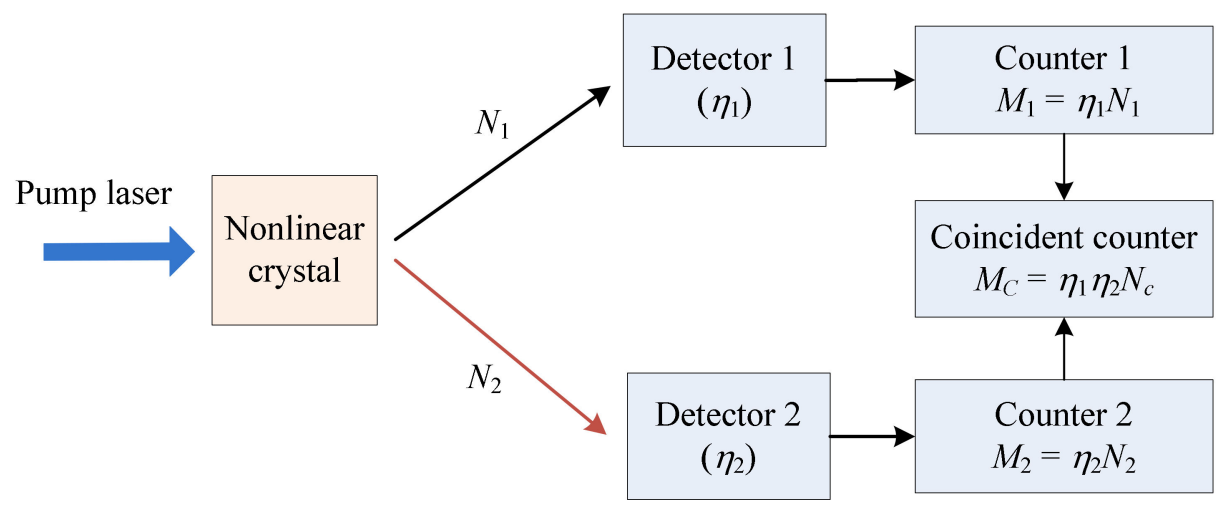

Figure 1. Spontaneous parametric down-conversion (SPDC)-based absolute calibration of detection efficiencies. 
SPDC satisfies energy conservation:

$$
v_{p}=v_{1}+v_{2}
$$

and momentum conservation

$$
k_{p}=k_{1}+k_{2} \text {. }
$$

where $v^{\prime}$ s and $k^{\prime}$ s are frequencies and wave vectors of photons, and the subscript $p, 1$ and 2 denote pumping and correlated photons, respectively.

Equation (1) defines the spectral range of correlated photons. Theoretically, SPDC will generate broadband photons with all frequencies $v_{1}$ and $v_{2}$ that satisfy Equation (1). The frequencies of correlated photons may extend from pump frequency to far infrared with a suitable choice of non-linear crystal, and will be limited by intrinsic properties of crystal such as spectral absorption. Under practical circumstances, down-conversion efficiency is usually the critical factor that defines a usable spectral range with sufficient photon rate.

Equation (2) decides the efficiency of SPDC, as well as exit angles and polarizations of correlated photons. By means of fine adjustment of incident angle, polarization of laser beam, and optical axis direction and birefringence properties of the crystal, correlated photons generated along the laser path inside the crystal will have the same phase when exiting the crystal. This phase matching condition needs an iterative optimization to balance down-conversion efficiency, spectral range and exit angles of correlated photons. As a consequence, only a limited spectral range, typically a few tens to a few hundred nanometers, is usable for a certain laser-crystal configuration. In order to cover a spectrum of 380-2500 nm for calibration of solar observing detectors, seven barium metaborate, $\beta-\mathrm{BaB}_{2} \mathrm{O}_{4}$ ), (BBO) crystals pumped by lasers of three wavelengths generate correlated photons with required spectral rates, polarization and exit angles, as shown in Figure 2. BBO is an optical crystal with high nonlinear coefficient and transmittance at 190-3500 nm and widely used as a media for optical frequency conversion.

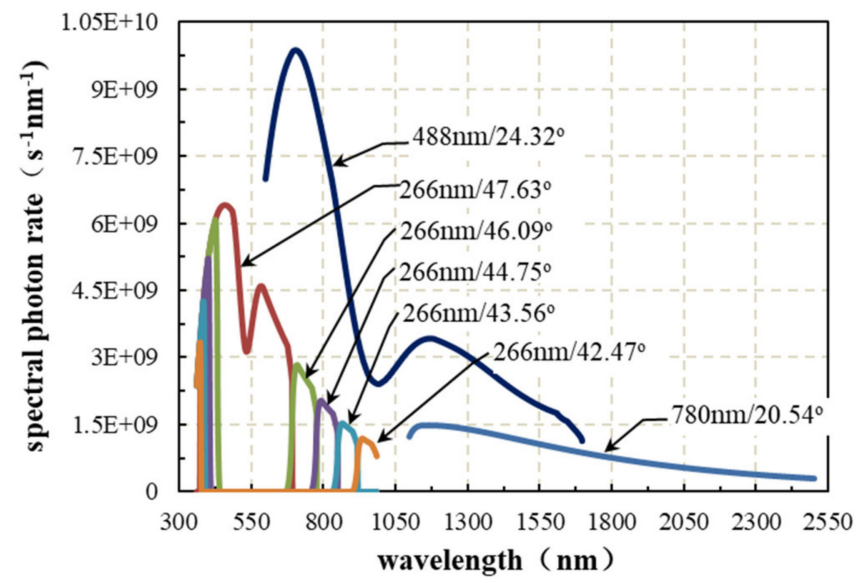

Figure 2. Spectral photon rates of correlated photons generated in seven barium metaborate, $\beta-\mathrm{BaB}_{2} \mathrm{O}_{4}$ ), (BBO) crystals pumped by three lasers at 266,488 and $780 \mathrm{~nm}$. Phase matching conditions are optimized to cover a spectrum of $380-2500 \mathrm{~nm}$ with photon rates higher than $10^{8} \mathrm{~s}^{-1} \mathrm{~nm}^{-1}$ and photon exit angles less than $6^{\circ}$. Numbers in legends represent pump wavelength/phase-matching angle.

As shown in Figure 1, two single photon detectors receive correlated photons and their output electronic pulses are registered with counters. The count rates, i.e., the numbers of electronic pulses generated in one second, will be

$$
\begin{aligned}
& M_{1}=\eta_{1} N_{1} \\
& M_{2}=\eta_{2} N_{2}
\end{aligned}
$$


where $M^{\prime}$ s are count rates of opto-electronic pulses, $\eta$ 's are quantum efficiencies of the two detection channels, and N's are correlated photon rates, i.e., numbers of correlated photons entering detection channels in one second. A coincidence counter gives coincident rate, which is the number of electronic pulses simultaneously produced by the two channels,

$$
M_{C}=\eta_{1} \eta_{2} N_{C}
$$

where $N_{C}$ is the rate of photons that simultaneously reach the two channels. The correlation of photon pairs guarantees $N_{1}=N_{2}=N_{C}$, therefore the absolute quantum efficiencies of two channels will be

$$
\begin{aligned}
& \eta_{1}=M_{C} / M_{2} \\
& \eta_{2}=M_{C} / M_{1}
\end{aligned}
$$

Equation (5) is the measurement equation for absolute detector calibration with SPDC. Traceability to an external radiometric standard is not required. $\eta$ 's are detection efficiencies of the entire measuring channels, from light entrance to counter output, including transmittance, reflectance and scattering along the optical path, quantum efficiencies of single photon detectors, as well as gains of electronic circuits. Consequently, the calibration is systematic and $\eta^{\prime}$ 's are actually absolute responsivities of the system.

Any degradation that occurs in optical or electronic components will be automatically reflected in detection efficiencies. For example, assuming detection efficiencies of the two channels changed to $\eta_{1} \tau_{1}$ and $\eta_{1} \tau_{2}$ due to degradations characterized by $\tau_{1}$ and $\tau_{2}$; count rates in Equation (3) will be $M_{1}=\eta_{1} \tau_{1} N_{1}$ and $M_{2}=\eta_{2} \tau_{2} N_{2}$, respectively. The coincidence count rate in Equation (4) will then be $M_{C}=\eta_{1} \tau_{1} \eta_{2} \tau_{2} N_{C}$. Without any modification, Equation (5) will give the changed absolute detection efficiencies as $\eta_{1}^{\prime}=M_{C} / M_{2}=\eta_{1} \tau_{1}$ and $\eta_{2}^{\prime}=M_{C} / M_{1}=\eta_{2} \tau_{2}$, given the correlation $N_{1}=N_{2}=N_{C}$. Therefore SPDC-based calibration can always provide the current and absolute responsivity which includes all changes in detection system. There is no need for monitoring or correction for degradation. In this sense the system with SPDC calibration is insensitive to degradation, which makes it a promising option for a space benchmark.

Since the 1980s many experiments [16-26] validated the feasibility for absolute calibration of detectors based on SPDC, and the reported uncertainties are about $2 \%-0.2 \%$ [18-25], which are comparable to current radiometric standards. The advancement of this technique prompts a redefinition of candela, one of the SI base units, with quantum candela $[27,28]$.

With known detection efficiency, a photon detector can measure rates of correlated photons. Taking advantage of correlation between channel 1 and 2 in Figure 1, i.e., $N_{1}=N_{2}$, we may obtain the absolute photon rate in channel 2 by measuring its companion photon rate in channel 1 . This feature is critical for shortwave infrared spectrum, for example wavelengths longer than $1700 \mathrm{~nm}$, where a single photon detector is less mature for space applications and an analog detector will be used for solar observation, as discussed in next section.

\section{Design of a SPDC-calibrated Solar Spectral Radiometer}

Solar radiation is the dominant incoming energy source of the Earth system and different spectral components of solar radiation play different roles in Earth's climate change. Since 1978 space sensors were deployed to make continuous observation of total and spectral irradiance of solar radiation [29]. Data discrepancies of a few percent between space missions [30,31] highlight the fact that the accuracy of onboard SI traceability remains a challenge to fulfill the scientific goal of space benchmarking.

In comparison with the effort that traces the solar spectral irradiance to optical power measured by an onboard electric substitute radiometer [32], we suggest a tracing chain that links solar spectral irradiance to photon rate and Planck's constant, by means of SPDC-based detector calibration described in the previous section. 
Figure 3 shows the prototype design of a solar spectral irradiance radiometer planned for space benchmarking at 380-2500 $\mathrm{nm}$. Incorporated with internal SPDC calibration, the instrument has two working modes, i.e., calibration and observation modes.

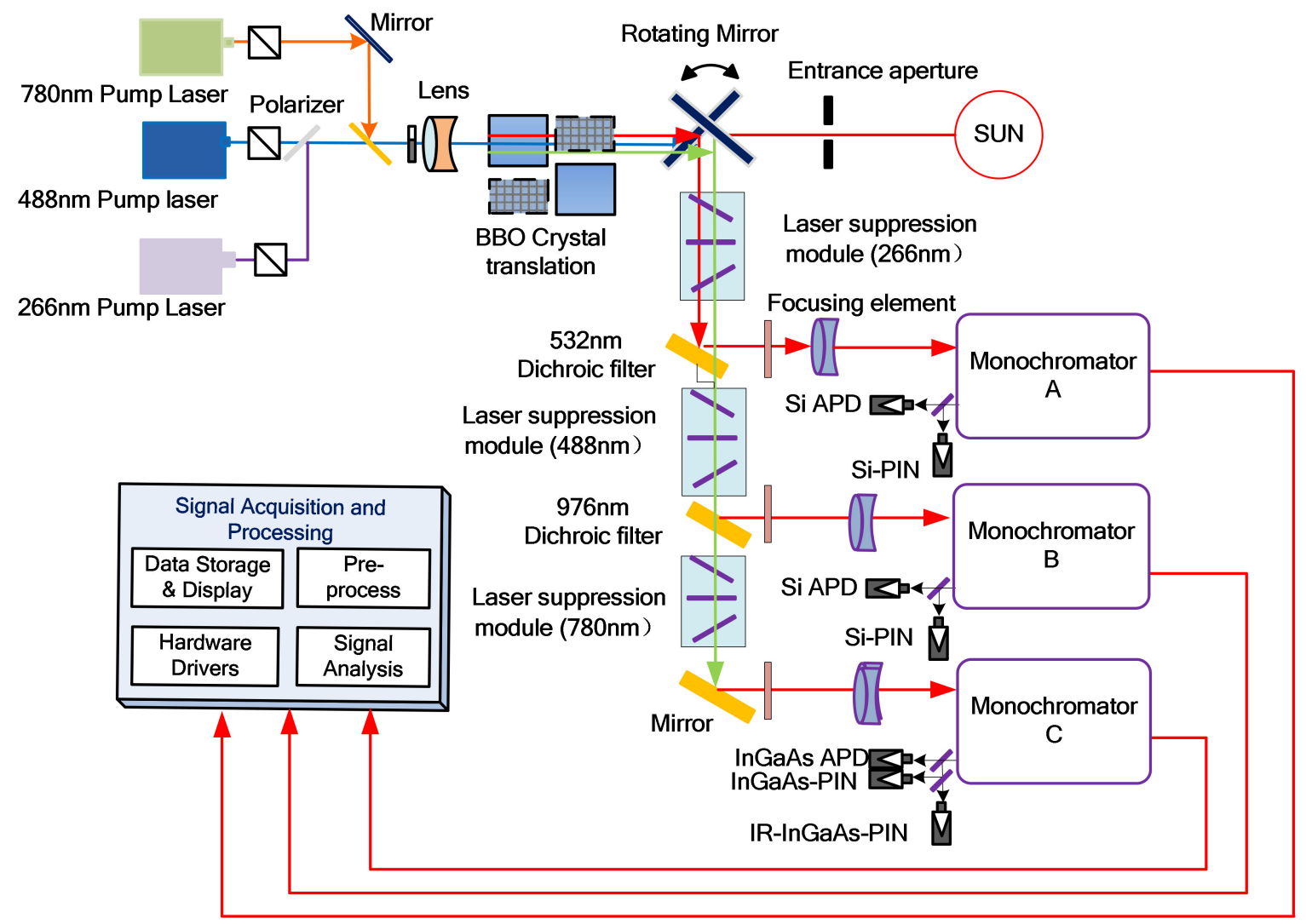

Figure 3. Schematic of a solar spectral irradiance radiometer calibrated with SPDC. Note: avalanche photodiode (APD).

In calibration mode correlated photons are generated with a suite of three lasers and seven BBO crystals as depicted in Figure 2. Continuous-wave lasers with output power of 50-100 mW are currently chosen to balance down-conversion efficiency, damaging threshold of crystal and overall power consumption and weight. Precise measurement of laser output power is not required according to the SPDC calibration principle. All lasers are equipped with feedback controlling modules to stabilize the output power within $0.1 \% / \mathrm{min}$ and ensure accuracy in calibration mode.

Crystals are installed in a temperature stabilized container and translated by an electric motor to match laser and crystal with specified phase-matching conditions. Correlated photons are directed by a rotating mirror into middle optics before finally reaching $\mathrm{Si}$ and InGaAs avalanche photodiodes (APDs). In middle optics, band-pass filters suppress residual pump photons by about 9 orders of magnitude. The laser suppression modules are designed for "long pass". The first suppression module blocks light below a threshold wavelength, e.g., $350 \mathrm{~nm}$ in the current system, so the pumping beam of $266 \mathrm{~nm}$ is removed, and correlated photons with wavelength $>350 \mathrm{~nm}$ pass through. Similarly, the second suppression module removes residual pumping photons of $488 \mathrm{~nm}$, and the third $780 \mathrm{~nm}$. In each suppression module correlated photons with wavelength $>$ threshold wavelength will pass through. Beam splitters separate correlated photon pairs and direct them into two monochromators with wavelengths settings satisfying Equation (1). Spectral resolution is currently configured as $3 \mathrm{~nm}$ for 380-1000 $\mathrm{nm}$ and $8 \mathrm{~nm}$ for 1000-2500 nm spectrum, respectively.

Absolute detection efficiencies of the entire channel are calibrated by the method described in Section 2. APDs are then used as absolute detectors to measure photon rates from the crystal. Since 
currently no APD is commercially available for wavelength larger than $1700 \mathrm{~nm}$, the photon rate at $1700-2500 \mathrm{~nm}$ is measured by measuring its correlated photon rate at 1441-1134 nm with a pumping wavelength of $780 \mathrm{~nm}$.

The correlated photon stream with a known rate can then be used as an absolute light source, since its absolute flux can be calculated as $\Phi=N h v$, where $N$ is photon rate, $h$ is Planck's constant, and $v$ is frequency. Analog photodiodes are calibrated against correlated photon flux and turn into absolute power detectors for a solar radiation measurement in observation mode.

In observation mode, a technically mature design of a spectroradiometer is adopted for good stability and repeatability. Solar rays passing an entrance aperture of $0.2 \times 7 \mathrm{~mm}$ are directed by the same rotating mirror into the same middle optics and monochromators as in the calibration mode. Instead of single photon detectors, Si, InGaAs and IR-InGaAs photodiodes are used in observation mode to measure absolute power of solar radiation at 380-960 nm, 900-1700 nm and 1600-2500 nm, respectively.

The solar spectral irradiance is then calculated with measured solar flux and known area of entrance aperture. The measurement procedure forms a traceability chain illustrated in the left part of Figure 4. As a comparison, a traceability chain based on electrical substitution radiometer (ESR), which is adopted in international space benchmarks $[1,9,10]$, is also shown in Figure 4. ESR-based measurement traces solar irradiance to electrical heating power of an internal absorptive cavity, and finally to electrical current and resistance. Its reliability benefits from a long development history over a century [32] and space application experiences in the past 20 years [9,10]. However inevitable decay of critical hardware such as change of cavity absorptance [1] remains a potential threat to ESR accuracy and long-term stability in space. SPDC-based measurement traces solar irradiance to photon counts and Planck's constant. Its validity relies on a physical effect that is independent of operation platforms and insensitive to decays in the system. The authors recently reported a sensitivity of less than $0.3 \%$ to a simulated system efficiency degradation of $61 \%$ in radiance measurements [26]. Before building a benchmark with unprecedented accuracy, thorough evaluations of uncertainties are demanded for single photon detection techniques and their newly developed applications in absolute radiometry, as outlined in next section.
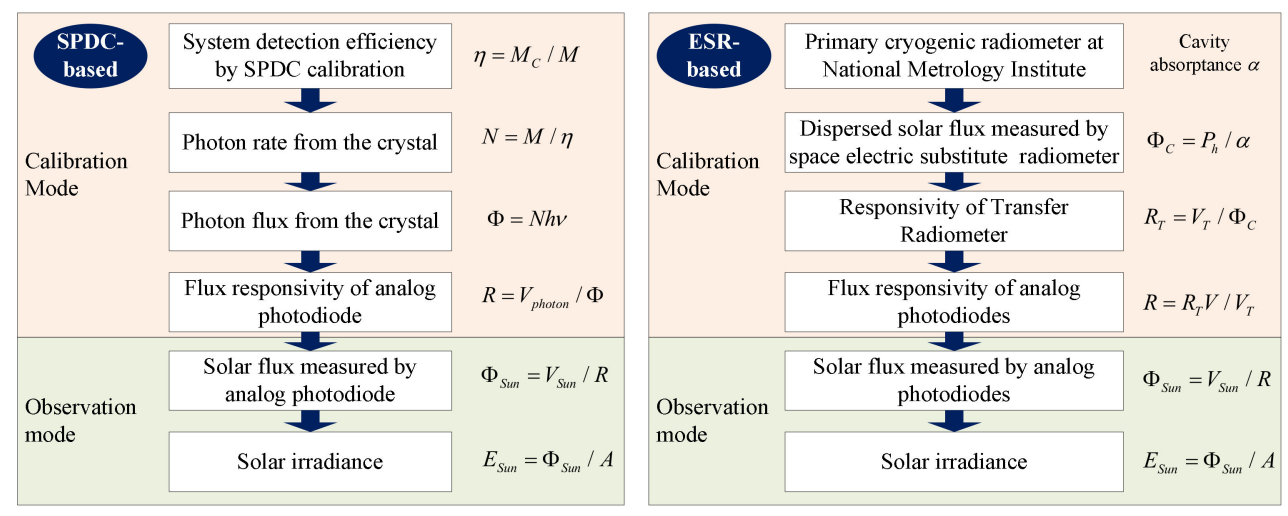

Figure 4. Traceability chain for solar spectral irradiance measurement: (left) SPDC-based, and (right) electrical substitution radiometer (ESR)-based [1].

\section{An Initial Evaluation of Uncertainty Budget}

SPDC-based radiometry is theoretically absolute and SI traceable. However, imperfect performances of optical and electronic components and the resultant errors will influence the systematic accuracy under practical circumstances. Evaluation and verification of measuring uncertainty at the highest level of accuracy form a part of the challenging goal of the benchmark mission.

According to the traceability route shown in Figure 4, uncertainty of solar irradiance measurement arises from both calibration and observation modes. In calibration mode, the accuracy of detection efficiency $\eta$ is solely decided by the accuracy of three counts of $M^{\prime}$ 's, i.e., counts of correlated channels 
and their coincidence, as shown in Equations (1)-(3). Properties of single photon detectors such as dark count, dead time and after-pulsing will introduce counting error or limit response linearity at high photon rate. Accidental count will occur in the coincidence counter due to time jittering of a single photon detector and arrival time inconsistency of correlated photons. Counting uncertainties due to these factors can be measured and corrected to minimize their contribution to the system uncertainty budget [18,23-26].

In calibration mode absolute responsivities of analog photodiodes are calibrated against correlated photon flux that is measured by single photon detectors. This transfer process consists of spectral scans by both detectors at correlated wavelengths that meet Equation (1). Photon flux will be measured at about 400 wavelengths over the working spectrum of $380 \mathrm{~nm}-2500 \mathrm{~nm}$ with current settings of spectral resolution. At each measured wavelength, single photon detectors and analog detectors make measurements alternately. Both detectors take time to receive sufficient photons to ensure an acceptable signal-to-noise-ratio. Measuring time at a single wavelength is about 6 to $10 \mathrm{~s}$ during which the laser power is stabilized within $0.1 \% / \mathrm{min}$ and the temperature of detectors is stabilized within $\pm 0.1^{\circ} \mathrm{C}$. The calibration may last 40-70 min and the time is expected to be reduced with negligible loss of accuracy by gradually reducing the number of calibration wavelengths to less than 100, with the help of an empirical model of the detector's spectral responsivity constructed from fine spectral scanning data.

For observation mode, uncertainties come mainly from a detector's nonlinearity and signal-to-noise ratio. Analog photodiodes are calibrated under a photon rate about $10^{9}$ photons/s as shown in Figure 2. While watching the Sun, the flux is about $10^{13}$ photons/s under current acceptance geometry and spectral bandwidth. Sunlight attenuation seems to be a straightforward solution to minimize photon rate difference and improve equivalence between calibration and observation mode. However, attenuation devices cannot be put in the common optical path and their degradation cannot be included in system detection efficiency measured in calibration mode. In this case attenuation ratio has to be monitored on orbit with a high precision, for example $0.1 \%$, to correct possible change due to solar exposure. To avoid the engineering complexity of solar attenuation, our current plan is to take full advantage of a photodiode's large dynamic range of 14 orders of magnitude [33], and cool analog detectors to a temperature about $-40{ }^{\circ} \mathrm{C}$ to improve response linearity and signal-to-noise-ratio under a weak photon rate. An empirical model for non-linearity correction can be constructed by precise measurement of the linearity factor as performed in metrology labs [34].

Stray light is one of the main sources of uncertainty both in calibration and observation mode. Residual pumping photon rate is about $10^{9}$ higher than that of correlated photons, and must be suppressed and separated from the correlated photons by spatial, spectral and polarization methods [26]. Scattering of correlated photons and solar photons at optical surfaces will result in background counts or background electric current, as well as accidental coincidence counts. Background count can be measured by switching off the SPDC effect with a $\lambda / 2$ wave plate [26], and background current can be characterized by blocking the incident solar light with a shutter.

Since the radiometer is still under fabrication, experimental test and evaluation of uncertainty for solar irradiance measurement are currently unavailable. The final measurement equation with corrections mentioned above will become more complicated than what is shown in Figure 4 . For example, the detection efficiency of channel 1 will be

$$
\eta_{1}=\frac{M_{C}-M_{C A}-M_{C B}}{M_{2}-M_{2 B}} \frac{1}{\alpha \cdot \beta}
$$

where $M_{C A}$ and $M_{C B}$ are counts of accidental and background coincidence, respectively. $M_{2 B}$ is the background count of channel 2. $\alpha$ and $\beta$ are correction factors for dead time of a single photon detector and lost count of coincidence, respectively.

Another important source of uncertainty comes from the transmittance of crystals which constitutes a part of detection efficiency obtained in calibration mode. As shown in Figure 3, the crystal is not 
in the optical path in observation mode and its transmittance must be determined and excluded for irradiance measurement. The solar irradiance at wavelength $\lambda$ will then be calculated by

$$
\begin{aligned}
E(\lambda) & =\frac{1}{\tau_{\text {crystal }}(\lambda)} \cdot \frac{V_{\text {sun }}(\lambda)}{R(\lambda) \cdot A} \\
& =\frac{1}{\tau_{\text {crystal }}(\lambda)} \cdot \frac{V_{\text {sun }}(\lambda)}{V_{\text {photon }}(\lambda)} \frac{M_{1}(\lambda)}{\eta_{1}(\lambda) \cdot A} \frac{h c}{\lambda},
\end{aligned}
$$

where $\tau_{\text {crystal }}(\lambda)$ represents the transmittance of the crystal, $V_{\text {sun }}(\lambda)$ and $V_{\text {photon }}(\lambda)$ are outputs of the radiometer in observation and calibration modes, respectively. $R(\lambda), M_{1}(\lambda)$ and $\eta_{1}(\lambda)$ are absolute responsivity, photon count and detection efficiency obtained in calibration mode, respectively, as depicted in Figure 4 and Equation (6). $A$ is the area of entrance aperture. To minimize the uncertainty of transmittance due to internal absorption, scattering and surface reflection, the thickness of a crystal is reduced to $2 \mathrm{~mm}$ and its exit surface is antireflection coated. In addition, a spectral transmittance model will be built based on pre-flight transmittance measurement over the whole working spectrum. In-flight transmittance measurement will be performed at three pumping wavelengths by moving crystals in and out of the pumping beam, which will provide parameters for updating the transmittance model. Fundamental wavelengths of pump lasers such as 532 nm, $960 \mathrm{~nm}$ and $1560 \mathrm{~nm}$ are optional to densify the measurements.

Based on the traceability chain in Figure 4, Table 1 summarizes the uncertainty sources that are identified in the current measurement procedure. A combined uncertainty of about $0.35 \%$ is allocated to individual contributors, referring to authors' past work and applicable evaluation approaches developed in the past 30 years [19-26]. Uncertainties in Table 1 are averages over 380-2500 nm and generally increase with wavelength due to lower photon rate at longer wavelengths as shown in Figure 2. To the authors' knowledge, about half of the constituent uncertainties in Table 1 are achievable with current metrological techniques, while the other half demands significant improvement of measuring protocols as well as facilities.

Table 1. Estimated uncertainty budget for solar irradiance measurement.

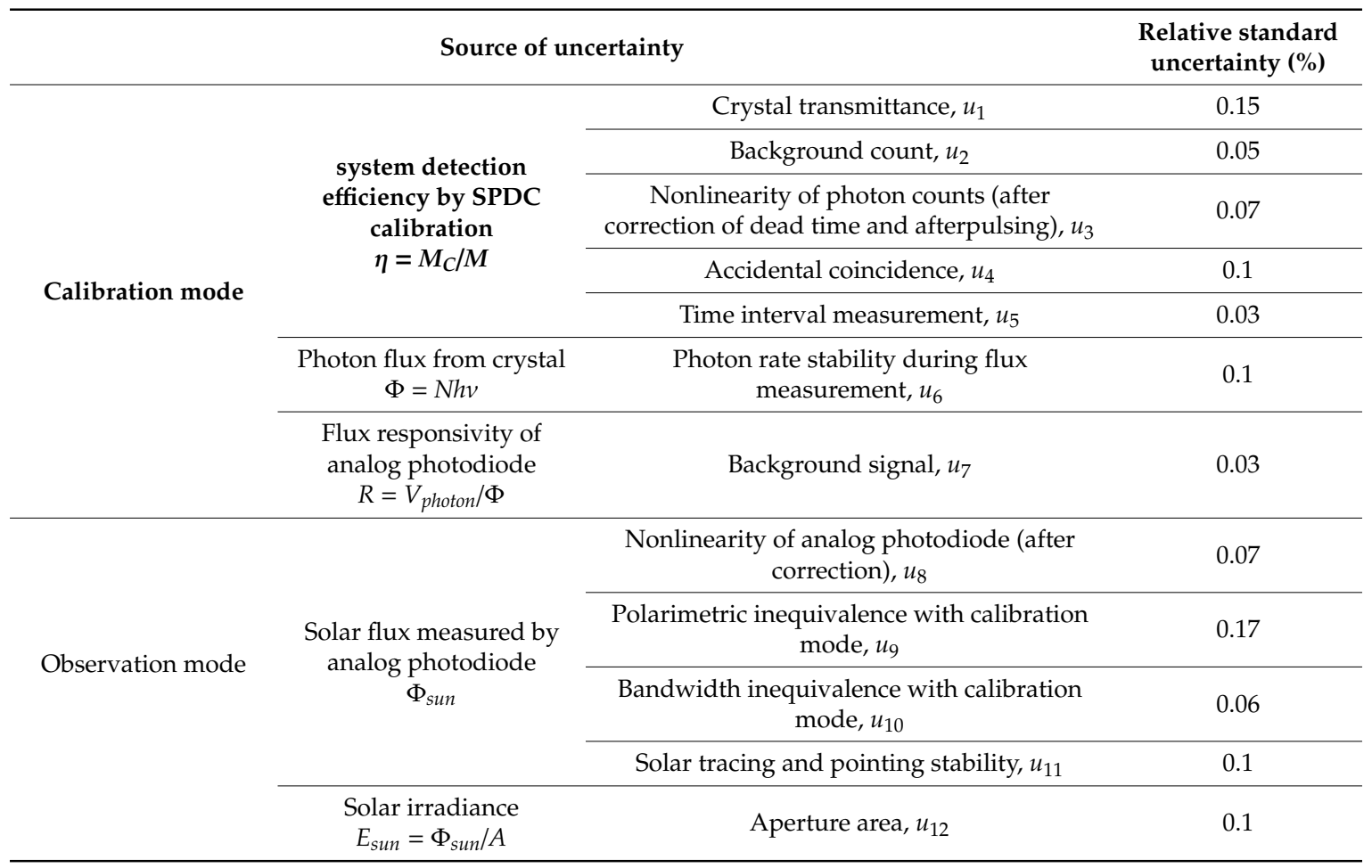


Table 1. Cont

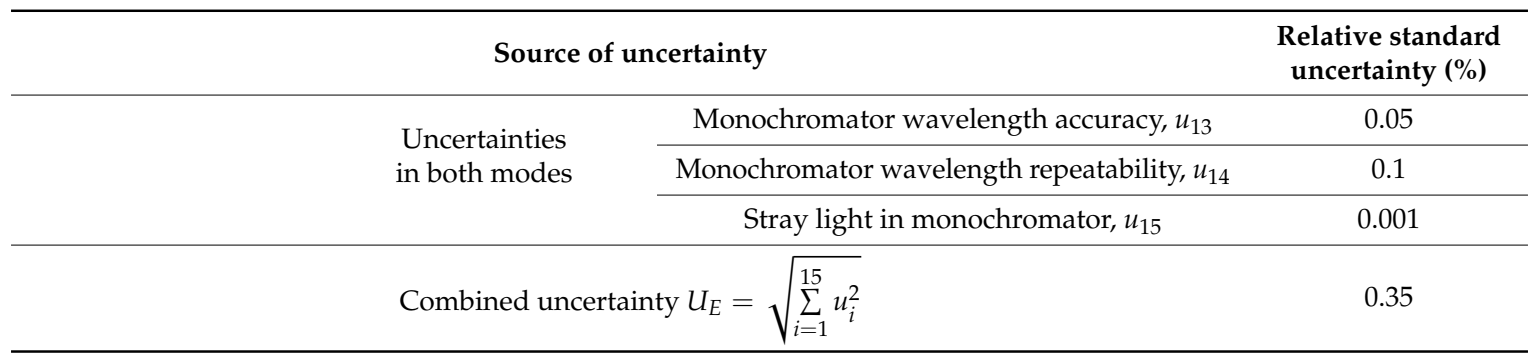

\section{Conclusions}

A solar spectral irradiance radiometer integrated with a quantum optical standard is proposed for a future space benchmark. Absolute solar irradiance is traceable to photon rates and Planck's constant by absolute calibration of detection efficiency with correlated photon pairs generated in spontaneous parametric down-conversion. The calibration is end-to-end and independent of external radiometric standards. A prototype of the SPDC-calibrated radiometer is currently under fabrication, supported by Chinese space benchmark project, and will be functional in mid-2022 for performance demonstration. This paper focuses on the working mechanism and conceptual description of system structure, especially the new traceability chain proposed for a space benchmark. Preliminary assessment of measurement uncertainty supports the rationality of this new mechanism for highly accurate radiometry. Performance improvement is expectable with advancement of single photon radiometry and maturity of new powerful devices [35], such as superconductive nanowire single photon detectors with significantly higher quantum efficiency and lower dark count. Many important engineering details such as the Sun pointing, space environment adaptability and retrieval algorithms for spectral irradiance have not yet been finalized and will be discussed in subsequent papers.

Author Contributions: Conceptualization, X.Z.; data curation, M.X. and Y.H.; formal analysis, M.X., W.Z., J.L. and Y.Y.; investigation, Y.H., J.L. and W.P.; methodology, X.Z.; software, Y.Y.; validation, J.L. and W.P.; visualization, W.Z.; Writing-original draft, X.Z. All authors have read and agreed to the published version of the manuscript.

Funding: This research and the APC were funded by Ministry of Science and Technology of China under grant No. 2018YFB0504602.

Acknowledgments: The authors acknowledge financial support of Chinese National Key Research and Development project under grant No. 2018YFB0504602. We also appreciate the helpful discussions with Naimeng Lu at the Chinese National Satellite Meteorological Center and Chuanrong Li at Aerospace Information Research Institute, Chinese Academy of Sciences.

Conflicts of Interest: The authors declare no conflict of interest.

\section{References}

1. Fox, N.; Aiken, J.; Barnett, J.; Briottet, X.; Carvell, R.; Frohlich, C.; Groom, S.B.; Hagolle, O.; Haigh, J.D.; Kieffer, H.H.; et al. Traceable Radiometry Underpinning Terrestrial- and Helio-Studies (TRUTHS). Adv. Space Res. 2003, 32, 2253-2261. [CrossRef]

2. Anderson, J.; Dykema, J.; Goody, R.; Hu, H.; Kirk-Davidoff, D. Absolute, spectrally-resolved, thermal radiance: A benchmark for climate monitoring from space. J. Quant. Spectrosc. Radiat. Transf. 2004, 85, 367-383. [CrossRef]

3. Ohring, G.; Wielicki, B.; Spencer, R.; Emery, B.; Datla, R. Satellite instrument calibration for measuring global climate change. Bull. Am. Meteorol. Soc. 2005, 86, 1303-1314. [CrossRef]

4. Wielicki, B.; Young, D.; Mlynczak, M.; Thome, K.J.; Leroy, S.; Corliss, J.; Anderson, J.G.; Ao, C.O.; Bantges, R.; Best, F.; et al. Archieving climate change absolute accuracy in obit. Bull. Am. Meteorol. Soc. 2013, 94, 1519-1539. [CrossRef]

5. Dinguirard, M.; Slater, P.N. Calibration of Space-Multispectral imaging sensors: A review. Remote Sens. Environ. 1999, 68, 194-205. [CrossRef] 
6. Xiong, X.; Eplee, R.E., Jr.; Sun, J.; Patt, F.S.; Angal, A.; McClain, C.R. Characterization of MODIS and SeaWiFS Solar Diffuser On-orbit Degradation. In Earth Observing Systems XIV, Proceedings of the SPIE Optical Engineering + Applications, San Diego, CA, USA, 2-6 August 2009; James, J.B., Xiaoxiong, X., Xingfa, G., Eds.; SPIE: San Diego, CA, USA, 2009; Volume 7452, p. 74520Y.

7. Zhi, D.; Wei, W.; Zhang, Y.; Yu, T.; Pan, Y.; Sun, L.; Li, X.; Zheng, X. FengYun-3 B satellite medium resolution spectral imager visible on-board calibrator radiometric output degradation analysis. IEEE Trans. Geosci. Remote Sens. 2019, 57, 3240-3251. [CrossRef]

8. BenMoussa, A.; Gissot, S.; Schühle, U.; Del Zanna, G.; Auchère, F.; Mekaoui, S.; Jones, A.R.; Walton, D.; Eyles, C.J.; Thuillier, G.; et al. On-Orbit Degradation of Solar Instruments. Sol. Phys. 2013, 288, 389-434. [CrossRef]

9. Harder, J.; Lawrence, G.M.; Rottman, G.; Woods, T. Solar Spectral Irradiance Monitor (SIM). Metrologia 2000, 37, 415-418. [CrossRef]

10. Kopp, G.; Lawrence, G. The total irradiance monitor (TIM): Instrument calibration. Sol. Phys. 2005, 230, 91-109. [CrossRef]

11. Best, F.; Adler, D.; Ellington, S.; Thielman, D.; Revercomb, H. On-orbit Absolute Calibration of Temperature with Application to the CLARREO Mission. In Earth Observing Systems XIII, Proceedings of the Optical Engineering + Applications, San Diego, CA, USA, 10-14 August 2008; James, J.B., Jack, X., Eds.; SPIE: San Diego, CA, USA, 2008; Volume 7081, p. 70810O. [CrossRef]

12. Louisell, W.; Yariv, A.; Seigman, A. Quantum Fluctuations and Noise in Parametric Processes. I. Phys. Rev. 1961, 124, 1646-1654. [CrossRef]

13. Hong, C.; Mandel, L. Theory of parametric frequency down conversion of light. Phys. Rev. A 1985, 31, 2409-2418. [CrossRef] [PubMed]

14. Yuan, Z.; Bao, X.; Lu, C.; Zhang, J.; Peng, C.Z.; Pan, J.W. Entangled photons and quantum communication. Phys. Rep. 2010, 497,1-40. [CrossRef]

15. Polyakov, S.; Migdall, A. Quantum radiometry. J. Mod. Opt. 2009, 56, 1045-1052. [CrossRef]

16. Klyshko, D. Use of two-photon light for absolute calibration of photoelectric detectors. Sov. J. Quantum Electron. 1980, 10, 1112-1117. [CrossRef]

17. Malygin, A.; Penin, A.; Sergienko, A. Absolute calibration of the sensitivity of photodetectors using a biphotonic field. JETP Lett. 1981, 33, 477-480.

18. Kwiat, P.; Steinberg, A.; Chiao, R.; Eberhard, P.; Petroff, M. Absolute efficiency and time-response measurement of single-photon detectors. Appl. Opt. 1994, 33, 1844-1853. [CrossRef] [PubMed]

19. Brida, G.; Castelletto, S.; Degiovanni, I.; Genovese, M.; Novero, C.; Rastello, M.L. Towards an Uncertainty Budget in Quantum Efficiency Measurements with Parametric Fluorescence. Metrologia 2000, 37, 629-632. [CrossRef]

20. Migdall, A. Absolute Quantum efficiency measurement using correlated photons: Toward a measurement protocol. IEEE Trans. Instrum. Meas. 2001, 50, 478-481. [CrossRef]

21. Migdall, A.; Castelletto, S.; Degiovanni, I.; Rastello, M. Intercomparison of a correlated-photon-based method to measure detector quantum efficiency. Appl. Opt. 2002, 41, 2914-2922. [CrossRef]

22. Feng, Y.; Zheng, X.; Li, J.; Zhang, W. Research in absolute calibration of single photon detectors by means of correlated photons. Chin. Opt. Lett. 2006, 4, 315-317.

23. Ghazi-Bellouati, A.; Razeta, A.; Bastie, J.; Himbert, M.E. Detector calibration at INM using a correlated photons source. Eur. Phys. J. Appl. Phys. 2006, 35, 211-216. [CrossRef]

24. Ware, M.; Migdall, A.; Bienfang, J.; Polyakov, S. Calibrating photon-counting detectors to high accuracy: Background and deadtime issues. J. Mod. Opt. 2007, 54, 361-372. [CrossRef]

25. Li, J.; Zheng, X.; Lu, Y.; Zou, P.; Zhang, W. Quantum efficiency calibration of opto-electronic detector by means of correlated photons method. Chin. Opt. Lett. 2008, 6, 472-475.

26. Hu, Y.; Li, J.; Gao, D.; Zheng, X. Absolute radiance measurement based on correlated photons calibration. Eur. Phys. J. D 2020, 74, 8. [CrossRef]

27. Cheung, J.; Chunnilall, C.; Woolliams, E.; Fox, N.P.; Mountford, J.R.; Wang, J.; Thomas, P.J. The quantum candela: A re-definition of the standard units for optical radiation. J. Mod. Opt. 2007, 54, 373-396. [CrossRef]

28. Lemieux, S.; Giese, E.; Fickler, R.; Chekhova, M.V.; Boyd, R.W. A primary radiation standard based on quantum nonlinear optics. Nat. Phys. 2019, 15, 529-532. [CrossRef] 
29. Ermolli, I.; Matthes, K.; Dudok deWit, T.; Krivova, N.A.; Tourpali, K.; Weber, M.; Unruh, Y.C.; Gray, L.; Langematz, U.; Pilewskie, P.; et al. Recent variability of the solar spectral irradiance and its impact on climate modeling. Atmos. Chem. Phys. 2013, 13, 3945-3977. [CrossRef]

30. Harder, J.; Thuillier, G.; Richard, E.; Brown, S.W.; Lykke, K.R.; Snow, M.; Mcclintock, W.E.; Fontenla, J.M.; Woods, T.N.; Pilewskie, P. The SORCE SIM Solar Spectrum: Comparison with Recent Observations. Sol. Phys. 2010, 263, 3-24. [CrossRef]

31. Pagaran, J.; Harder, J.; Weber, M.; Floyd, L.; Burrows, J. Intercomparison of SCIAMACHY and SIM vis-IR irradiance over several solar rotational timescales. Astron. Astrophys. 2011, 528, A67. [CrossRef]

32. Parr, A. The candela and photometric and radiometric measurements. J. Res. Natl. Inst. Stand. Technol. 2001, 106, 151-186. [CrossRef]

33. Eppeldauer, G.; Hardis, J. Fourteen-decade photocurrent measurements with large-area silicon photodiodes at room temperature. Appl. Opt. 1991, 30, 3091-3099. [CrossRef] [PubMed]

34. Haapalinna, A.; Kübarsepp, T.; Kärhä, P.; Ikonen, E. Measurement of the absolute linearity of photodetectors with a diode laser. Meas. Sci. Technol. 1999, 10, 1075-1078. [CrossRef]

35. Eisaman, M.; Fan, J.; Migdall, A.; Polyakov, S. Invited Review Article: Single-photon sources and detectors. Rev. Sci. Instrum. 2011, 82, 134-202. [CrossRef] [PubMed]

(C) 2020 by the authors. Licensee MDPI, Basel, Switzerland. This article is an open access article distributed under the terms and conditions of the Creative Commons Attribution (CC BY) license (http://creativecommons.org/licenses/by/4.0/). 